\title{
Can artificial tears prevent Acanthamoeba keratitis? An in vitro approach
}

\author{
Angela Magnet ${ }^{1}$, Thiago Santos Gomes ${ }^{1,5}$, Carmen Pardinas ${ }^{1}$, Natalia Garcia de Blas ${ }^{1}$, Cruz Sadaba ${ }^{2}$, \\ Eugenia Carrillo ${ }^{4}$, Fernando Izquierdo ${ }^{1}$, José Manuel Benítez del Castillo ${ }^{3}$, Carolina Hurtado ${ }^{1}$, \\ Carmen del Aguila ${ }^{1}$ and Soledad Fenoy ${ }^{1 *}$
}

\begin{abstract}
Background: The use of contact lenses has increased in recent years as has the incidence of Dry Eye Syndrome, partly due to their use. Artificial tears are the most common treatment option. Since these changes can facilitate Acanthamoeba infection, the present study has been designed to evaluate the effect of three artificial tears treatments in the viability of Acanthamoeba genotype T4 trophozoites. Optava Fusion ${ }^{\mathrm{TM}}$, Oculotect ${ }^{\oplus}$, and Artelac ${ }^{\oplus}$ Splash were selected due to their formulation.

Methods: Viability was assessed using two staining methods, Trypan Blue stain and CTC stain at different time intervals (2, 4, 6, 8 and $24 \mathrm{~h}$ ). Trypan Blue viability was obtained by manual count with light microscopy while the CTC stain was determined using flow cytometry.

Results: Trypan Blue staining results demonstrated a decrease in viability for Optava Fusion ${ }^{\mathrm{TM}}$ and Artelac $^{\circledR}$ Splash during the first $4 \mathrm{~h}$ of incubation. After, this effect seems to lose strength. In the case of Oculotect ${ }^{\oplus}$, complete cell death was observed after 2 h. Using flow cytometry analysis, Optava Fusion ${ }^{\text {TM }}$ and Oculotect ${ }^{\oplus}$ exhibited the same effect observed with Trypan Blue staining. However, Artelac ${ }^{\circledR}$ Splash revealed decreasing cell respiratory activity after four hours, with no damage to the cell membrane.

Conclusions: The present study uses, for the first time, CTC stain analyzed by flow cytometry to establish Acanthamoeba viability demonstrating its usefulness and complementarity with the traditional stain, Trypan Blue. Artelac ${ }^{\circledR}$ Splash, with no preservatives, and Optava Fusion TM, with Purite ${ }^{\oplus}$, have not shown any useful amoebicidal activity. On the contrary, promising results presented by Ocultect ${ }^{\oplus}$, with BAK, open up a new possibility for Acanthamoeba keratitis prophylaxis and treatment although in vivo studies should be carried out.
\end{abstract}

Keywords: Acanthamoeba, Prophylaxis, Artificial tears, Preservatives, Amoebicidal effect

\section{Background}

Acanthamoeba spp. are one of the most commonly isolated amoebae in environmental samples. They have a cosmopolitan distribution and can act as both opportunistic and non-opportunistic pathogens [1]. These amoebae have been isolated from soil, dust, air, seawater, swimming pools, sewage, sediments, airconditioning units, domestic tap water, bottled water, dental treatment units, hospitals, dialysis apparatus, eyewash stations, contact lenses and their cases and

\footnotetext{
* Correspondence: sfenrod@ceu.es

'Facultad de Farmacia, Universidad San Pablo CEU, CEU Universities, Madrid,

Spain

Full list of author information is available at the end of the article
}

as contaminants in bacterial, yeast and mammalian cell cultures [2, 3]. Acanthamoeba species present two morphological stages in their life-cycle: a vegetative trophozoite stage, in which they are active and reproduce by binary fission, and a cyst stage that is resistant to environmentally adverse conditions $[4,5]$.

These free-living amoebae are typically harmless to humans, but in rare instances can cause severe infections. One of these infections, Acanthamoeba keratitis (AK), is an ulceration of the cornea which, if not treated promptly, can cause extensive ocular damage, leading to loss of vision acuity, blindness and possible enucleation $[4,6-8]$. 
In recent decades, there has been a remarkable rise in the number of diagnosed AK cases, mostly due to an increase in the number of contact lens (CL) wearers $[9,10]$. Most of these cases affect immunocompetent CL wearers and result from poor hygiene practices as well as the failure to comply with recommended cleaning and disinfection procedures, rinsing with tap water or homemade saline solutions, showering while wearing lenses and the extended use of disposable CL [11].

Contact lenses wear alone is frequently associated with symptoms of ocular irritation, including dryness, discomfort, soreness and tiredness [12]. Sometimes these signs of ocular surface impairment resemble dry eye conditions in non-lens wearers. Dry eye syndrome (DES) is a disorder of the tear film due to tear deficiency or excessive tear evaporation, which can cause damage to the interpalpebral ocular surface. It is also associated with symptoms of ocular discomfort, and contact lens dry eye is considered a sub-classification of this syndrome $[13,14]$.

The sole presence of a CL on the eye affects the nature of tear film dispersal. A reduction in the prelens tear film lipid layer and an increase in tear film evaporation are attributed to $\mathrm{CL}$ wear, resulting in the onset of dryness. Additionally, the disruption of the tear film by the CL may lead to compromised functional visual acuities, reduced wear time, and an increased risk of ocular surface desiccation, bacterial binding and infection [12]. These alterations in the corneal epithelium produced both by DES and CL wear, can create a possible entry point for ocular surface invasion [15]. For example, several in vivo studies indicate that corneal trauma is a prerequisite for AK, as animals with intact corneas (i.e. epithelial cells) do not develop this infection [5].

For the treatment of these symptoms, rewetting drops are traditionally the most common first-line option. However, technological advances have led to the development of artificial tear solutions, also known as lubricant eye drops, which mimic the tear film function and protect the ocular surface from dryness. Recently, several artificial tear solutions have been produced to reduce these symptoms. They account for at least $\$ 540$ million in annual sales globally and are currently the mainstay of therapy of DES due to their noninvasive nature and reduced side effect profile [12, 16, 17].

Moreover, these artificial tears are formulated with different preservative compounds that may present useful properties in preventing ocular infections. In this study, the potential amoebicidal effects of artificial tear solutions were evaluated for the first time in order to establish their usefulness in the prevention of AK.

\section{Methods}

\section{Acanthamoeba culture}

Acanthamoeba spp. strain USP-CR5-A35 genotype T4, which was originaly isolated form a Spanish keratitits patient, was used to study the effect of the artificial tears analyzed [18]. Axenic cultures were grown in PYG medium $(0.75 \%$ protease peptone, $0.75 \%$ yeast extract and $1.5 \%$ glucose with $40 \mu \mathrm{g}$ gentamicin per milliliter) at $28{ }^{\circ} \mathrm{C}$ without shaking. After $24 \mathrm{~h}$ of culture, the culture flask was incubated on ice for $5 \mathrm{~min}$ to favor amoebae de-attachment and then trophozoites were collected with the help of a pipette, washed twice with NEFF saline [19] and the concentration was adjusted in NEFF to $4 \times 10^{5}$ amoebae $/ \mathrm{ml}$.

\section{Tested artificial tears}

Three different commercially available artificial tears were chosen for this study due to the different types of preservatives (detergent or oxidative) or the lack of them (Artelac ${ }^{\ominus}$ Splash, Bausch \& Lomb, Berlin, Germany) in their formulation. Characteristics of the different tears are shown in Table 1. Benzalkonium chloride (BAK) which interrupts the lipid component of cell membranes acting as an antibiotic and amoebicidal) is a detergent found in Oculotect ${ }^{\oplus}$ (Laboratoires Alcon, Kaysersberg, France) [20]. Purite ${ }^{\oplus}$ (Allergan, Westport, Ireland) is a stabilized oxychloride complex with chlorine dioxide, chlorite and chlorate found in Optava Fusion $^{\mathrm{TM}}$ (Allergan, Westport, Ireland). When excited by light, they produce water, oxygen, sodium, and chlorine free radicals. These radicals interact with the pathogen membrane causing death. To our knowledge, Purite ${ }^{\circledR}$ has never been tested for Acanthamoeba.

\section{Effect of artificial tears against Acanthamoeba, culture and microscopic observation}

In T25 culture bottles, $2.5 \mathrm{ml}$ of the amoebae suspension (USP-CR5-A45 at $4 \times 10^{5}$ amoebae $/ \mathrm{ml}$ ) was cultured with $2.5 \mathrm{ml}$ of the artificial tears. Non-effect control was performed with NEFF saline. These cultures were incubated at $33{ }^{\circ} \mathrm{C}$ for $2 \mathrm{~h}, 4 \mathrm{~h}, 6 \mathrm{~h}$ and $8 \mathrm{~h}$ as previously described [20-22]. Before culture collection, the appearance of the Acanthamoeba trophozoites was checked under light microscopy at 400× (Nikon Eclipse TS100, Nikon Instruments Europe B.V.). Cultures were collected and washed with PBS by centrifugation at $1500 \times \mathrm{rpm}$ for $10 \mathrm{~min}$. After, amoebae were suspended in $1 \mathrm{ml}$ of PBS and divided into two aliquots for the viability test. Culture with the artificial tears was performed in triplicate for the four different incubation times. 
Table 1 Characteristics of the artificial tears studied and cytometry results are shown as viability percentage

\begin{tabular}{|c|c|c|c|c|c|c|}
\hline & \multirow[t]{2}{*}{ Components } & \multirow[t]{2}{*}{ Preservative } & \multicolumn{4}{|c|}{$\begin{array}{l}\% \text { viability (TB); \% viability (CTC); \% altered } \\
\text { morphology (CTC) }\end{array}$} \\
\hline & & & $2 \mathrm{~h}$ & $4 \mathrm{~h}$ & $6 \mathrm{~h}$ & $8 \mathrm{~h}$ \\
\hline $\begin{array}{l}\text { Oculotect }{ }^{\oplus} 50 \mathrm{mg} / \mathrm{ml} \\
\text { (Novartis) }\end{array}$ & Povidone K25; sodium hidroxide & $\begin{array}{l}\text { Benzalkonium } \\
\text { chloride (BAK) }\end{array}$ & $3.6 ; 4.9 ; 0$ & $1.8 ; 7.3 ; 0$ & $5.5 ; 1.5 ; 0$ & $2.5 ; 4.1 ; 0$ \\
\hline $\begin{array}{l}\text { Optava Fusion } \\
\text { (Allergan) }\end{array}$ & $\begin{array}{l}\text { Sodium hyaluronate; sodium carboxymethylcellulose; } \\
\text { glycerin; erythritol }\end{array}$ & Purite $^{\oplus}$ & $\begin{array}{l}44.3 ; 44.5 ; \\
16.6\end{array}$ & $\begin{array}{l}46.5 ; 26.7 ; \\
6.8\end{array}$ & $\begin{array}{l}79.0 ; 34.2 ; \\
19.8\end{array}$ & $\begin{array}{l}85.7 ; 31.0 ; \\
28.7\end{array}$ \\
\hline $\begin{array}{l}\text { Artelac }{ }^{\oplus} \text { Splash } \\
\text { (Bausch+Lomb) }\end{array}$ & $\begin{array}{l}\text { Sodium hyaluronate; sodium chloride; potassium } \\
\text { chloride; sodium phosphates }\end{array}$ & None & $\begin{array}{l}52.0 ; 58.8 \\
3.3\end{array}$ & $\begin{array}{l}58.0 ; 34.6 \\
12.6\end{array}$ & $\begin{array}{l}72.0 ; 52.8 ; \\
4.1\end{array}$ & $\begin{array}{l}81.5 ; 10.8 ; \\
10.9\end{array}$ \\
\hline
\end{tabular}

\section{Viability tests}

\section{Trypan blue stain}

Trypan Blue 0.4\% (BioWhittaker ${ }^{\bullet}$, Walkersville, USA) was used 1:1 with the amoebae suspension to establish Acanthamoeba viability after incubation with the different tears. Trypan Blue is a vital stain that colors only dead cells. Living amoebae have a refringent appearance under light microscopy while dead amoebae exhibit a blue color. Cells were counted using a BRAND ${ }^{\circ}$ counting chamber BLAUBRAND ${ }^{\circ}$ Neubauer pattern (Sigma-Aldrich, Merck KGaA, Darmstadt, Germany).

\section{Flow cytometry}

To establish Acanthamoeba viability, Bacstain-CTC Rapid Staining Kit (Dojindo,Kumamoto, Japan) was used to stain viable amoebae. CTC (5-cyano-2,3-ditolyl-tetrazolium chloride) is a stain used to measure bacterial viability. CTC is a soluble reagent which, when it interacts with the respiratory system, gains a proton and becomes an insoluble product, CTC formazan. This product is fluorescent, a characteristic that allows it to be used in flow cytometry. Kobayashi et al. [23] first described the use of this product for Acanthamoeba viability in a fluorometric assay.

Amoebae were cultured with CTC for $30 \mathrm{~min}$ and then washed and fixed with PBS-1\% formalin. Parasites were identified based on forward/side scatter values and a total of 10,000 events was obtained by FACScalibur cytometer using the CELLquest software. The fluorescence was evaluated in FL3 histograms. Cytometer files were analyzed using FlowJo software (Version 7.6.5, Tree Star, Ashland, USA).

\section{Results}

\section{Microscopic observation}

Cultures were observed using an inverted microscope at $\times 400$ magnification before the amoebae were collected. As shown in Fig. 1 morphological changes were observed after $8 \mathrm{~h}$. Amoebae in NEFF saline solution as well as in Artelac $^{\bullet}$ Splash presented the typical amoebae
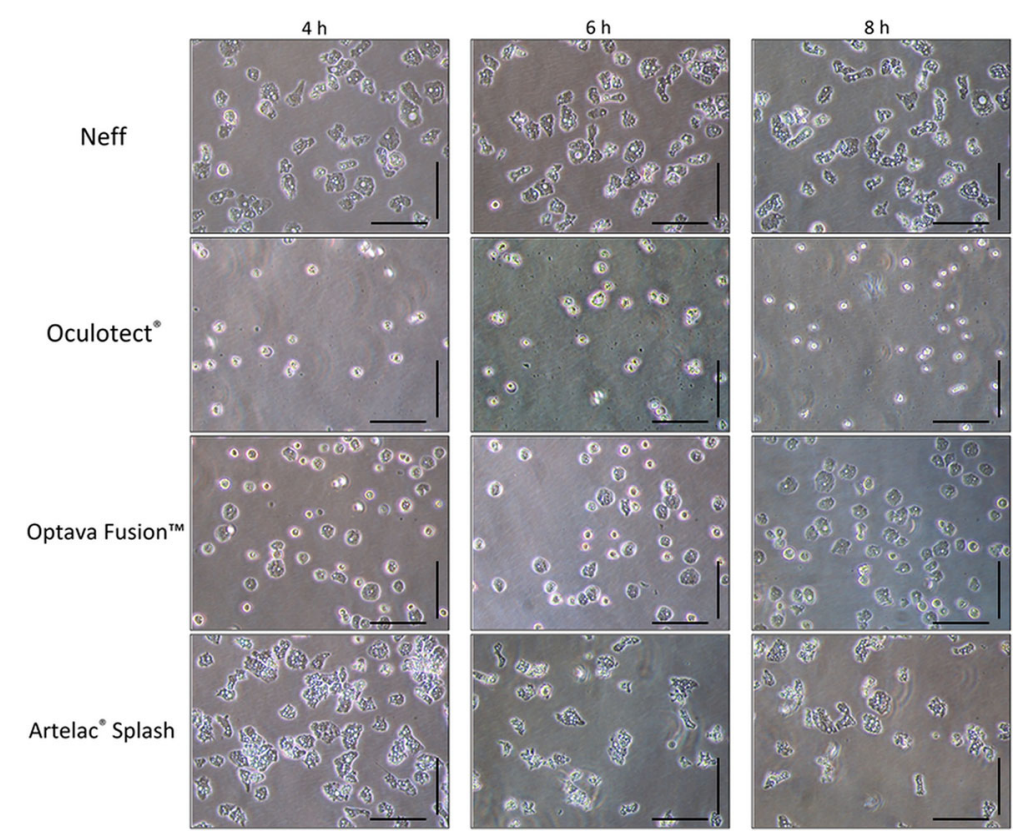

Fig. 1 Acanthamoeba culture appearance at 4, 6 and $8 \mathrm{~h}$ of incubation with the studied artificial tears. Scale-bars: $100 \mu \mathrm{m}$ 
morphology attached to the culture flask with acanthopodia projecting and vacuolated cytoplasm, while amoebae in Optava Fusion ${ }^{\mathrm{Tm}}$ were detached and rounded. In the culture bottle containing Oculotect ${ }^{\circ}$, only cellular debris was observed (Fig. 1).

\section{Viability tests \\ Trypan blue stain}

Viability studied using Trypan Blue showed a decrease in the case of Optava Fusion ${ }^{\text {Tx }}$ and Artelac ${ }^{\circ}$ Splash after the first $4 \mathrm{~h}$. Nevertheless, after $8 \mathrm{~h}$, recuperation of the amoebae was observed in both tears, amoebae concentration being similar to that of the NEFF control. In the case of Oculotect ${ }^{\circ}$ Acanthamoeba trophozoites were not detected after $2 \mathrm{~h}$ of incubation.

\section{Flow cytometry}

The flow cytometric analyses identified one population of parasites based on forward/side scatter values (R1) (Fig. 2). A second population of altered smaller trophozoites, R2, was observed after incubation with the artificial tears (Fig. 2), except for Oculotect ${ }^{\circ}$ incubation where only an R1 population was observed for all the incubation times tested. After gating on the basis of forward/side scatter values, the quantification of the relative fluorescence intensity was determined for both populations of each sample. Based on the histogram representing the viability of standard trophozoites, an area (M1) was determined for the measurement of the percentage fluorescent amoebae in all samples. For the NEFF control, the percentage of viable amoebae decreased from $74.9 \%$ to $45.6 \%$ in 8 h (Table 1). After two hours' treatment with Artelac ${ }^{\circ}$ Splash and Optava Fusion $^{\text {Tw }}$ a reduction of the viable population in R1 to 58.8 and $44.5 \%$ respectively, was observed. After 8 h, viability was 10.8 and $31.0 \%$, respectively, which means a 4.22-fold and 1.47-fold increase with regard to NEFF. The percentage population of amoebae in R2 increased from 3.3 to $10.9 \%$ in $8 \mathrm{~h}$ for Artelac ${ }^{\circ}$ Splash, and from 16.6 to $28.8 \%$ for Optava Fusion ${ }^{\mathrm{nw}}$. Treatment with Oculotect for $2 \mathrm{~h}$ reduced the population of fluorescent amoebae to $4.9 \%, 15.35$-times with regard to NEFF. The effect of Oculotect $^{\circ}$ was constant from the beginning and the percentage of viable amoebae remained similar at $8 \mathrm{~h}$ of incubation (4.1\%) (Table 1).

\section{Discussion}

In the present study, three artificial tear brands were selected according to the preservative agents (detergent or oxidative) or the lack of them to study their possible amoebicidal activity against trophozoites, as this is the initial state of the infection.

To study the viability of Acanthamoeba trophozoites after incubation with these tears, two different methods were used. The more traditional one, Trypan Blue stain, allows the differentiation of amoebae with the altered cell membrane and thus, identification of the non-viable ones. The second method used CTC stain to measure mitochondrial activity to determine the viability of the amoebae. This stain was developed for bacterial viability and then, adapted for Acanthamoeba in fluorometry by Kobayashi et al. [23]. In the present study, CTC stain for Acanthamoeba viability was used with flow cytometry for the first time.

One of the artificial tears tested using these two methods was Optava Fusion ${ }^{\mathrm{Tx}}$. It contains Purite, which is a soft oxidative preservative agent with demonstrated antimicrobial activity (bacteria, viruses and fungi), which dissociates on the eye surface in a photolytic reaction [24]. Contact of Acanthamoeba trophozoites with this artificial tear produced a slight decrease in the mitochondrial activity of amoebic cells (R1) during the incubation interval. However, a population of morphologically altered amoebae was observed which increased to $28.8 \%$ after eight hours (R2). If viability is considered as the sum of the two morphologies, results were similar to those obtained with Trypan Blue stain. In consequence, evaluation of both parameters, membrane integrity, and cell respiratory activity, seems to be crucial in the evaluation of the effectiveness of artificial tears and potential anti-amoebic compounds.

A further artificial tear evaluated was Artelac ${ }^{\circ}$ Splash, whose formula does not contain any preservatives. Analysis of this artificial tear with Trypan Blue staining seemed to produce the same effect as the one observed in the presence of Optava Fusion ${ }^{\text {Tu }}$, demonstrating an apparent decrease in amoebae viability after two hours but a discrete increase for longer periods. However, analysis with flow cytometry and CTC staining revealed decreasing cell respiratory activity in the studied time intervals without morphological change (R1 and R2). Additionally, both Optava Fusion ${ }^{\mathrm{rm}}$ and Artelac ${ }^{\circ}$ Splash contain sodium hyaluronate in their composition, a salt that has been proved to be a stimulator of proliferation of the corneal epithelium [25], so it could be also stimulating amoebic growth.

In the case of the artificial tear Oculotect ${ }^{\circ}$, the preservative agent used is BAK, a known antibiotic and amoebicidal compound [20]. This study demonstrated that BAK, in its commercially available formulation, maintains good amoebicidal activity despite the other components of the artificial tear and even when the tear is diluted 1:1. This amoebicidal effect was observed from the first hours of incubation and it maintained its effectiveness during all the time intervals evaluated with both viability techniques used. A decrease in the number of amoebic cells with Trypan Blue and low mitochondrial activity was observed using CTC. 
$2 \mathrm{~h}$
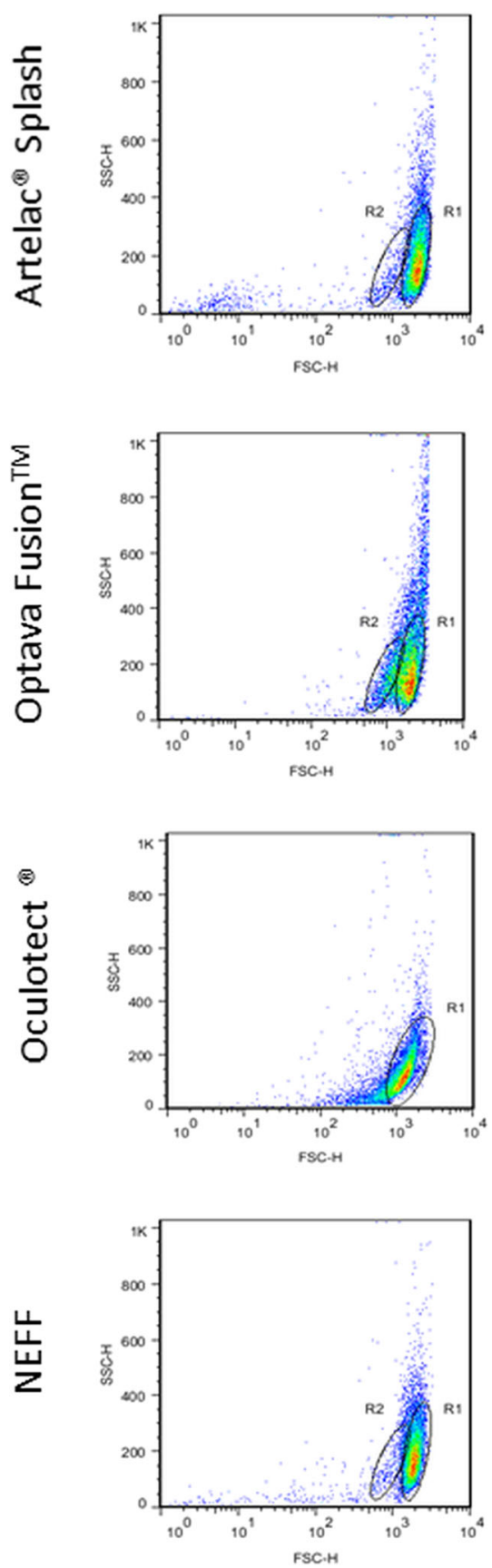

$8 \mathrm{~h}$
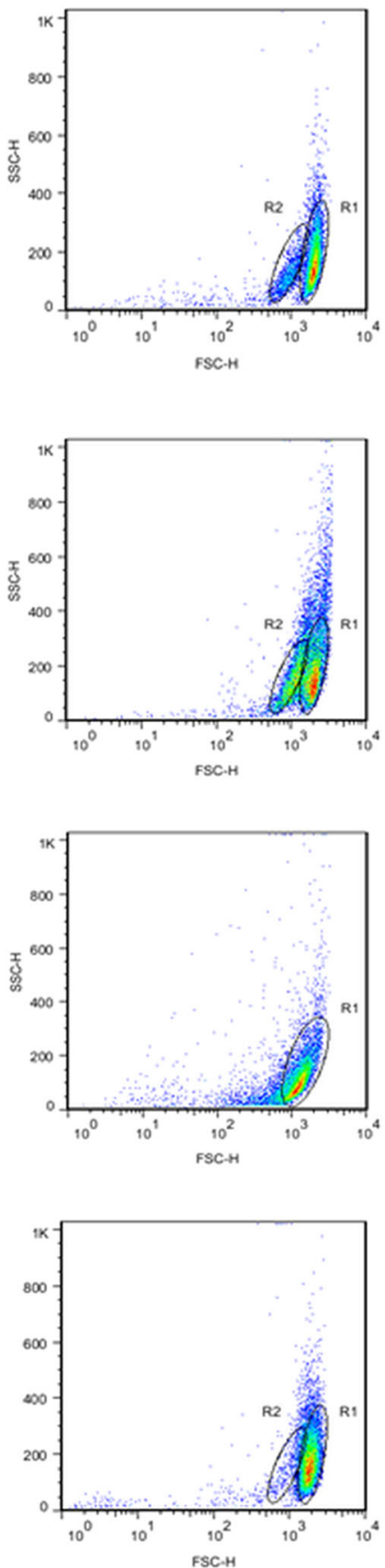

Fig. 2 Histogram statistic of the CTC viability assay of Artelac ${ }^{\circledR}$ Splash, Optava Fusion ${ }^{\text {TM, }}$, Oculotect ${ }^{\circledR}$ and NEFF by flow cytometry at 2 h, 4 h, 6 h and $8 \mathrm{~h}$ incubation. R1: normal amoebae population; R2: altered smaller amoebae trophozoites

In view of these results, we must highlight the importance of the data obtained with both methods used to investigate the viability of Acanthamoeba trophozoites. Trypan Blue staining and flow cytometry provide quite similar information for viability analysis, but they evaluate different characteristics, in such a way that the data they reveal are not exclusionary but complementary. As was observed with Artelac ${ }^{\circ}$ Splash, some cells can diminish their mitochondrial activity gradually while maintaining the integrity of the plasmatic membrane, a characteristic that can be interpreted differently by these two methods if they are used separately. Our results 
demonstrate that conventional viability staining with Trypan Blue is a useful method. Nevertheless, flow cytometry analysis with CTC staining can be helpful in eliminating observer bias due to its automated feature and this prevents possible misevaluations caused by overexposure to Trypan Blue.

Finally, in this study, only Oculotect ${ }^{\circ}$ was demonstrated to have an important and durable amoebicidal effect on Acanthamoeba trophozoites. However, we must be aware of the possible surface toxicity that BAK can produce on the cornea epithelium with frequent or extended use. This characteristic means that it is not advisable to use this artificial tear concomitantly with soft lenses. Hard contact lenses adsorb a small amount of this preservative and release a significant percentage of it during a regular washout procedure, approaching the upper safety limit, but soft contact lenses present a high adsorption of this substance. Such a property could allow the accumulation of BAK in the cornea epithelium, producing adverse effects [20]. Despite BAK's toxicity, the promising results showed by Oculotect ${ }^{\circ}$, its use as a PrEP (pre-exposure prophylaxis) strategy before eye surgery should be considered. Nevertheless, studies in corneal models or even in patients are needed to establish the in vivo amoebicidal effect of this kind of artificial tear formulation.

\section{Conclusions}

To our knowledge, the present study uses for the first time CTC stain analyzed by flow cytometry to establish Acanthamoeba viability demonstrating its usefulness and complementarity with the traditional stain, Trypan Blue. Artelac ${ }^{\circ}$ Splash, with no preservatives, and Optava Fusion $^{\mathrm{Tm}}$, with Purite $^{\ominus}$, have not shown any amoebicidal activity. On the contrary, promising results shown by Ocultect ${ }^{\circ}$, with BAK, open up a new possibility of Acanthamoeba keratitis prophylaxis and treatment, although in vivo studies should be carried out.

\section{Abbreviations}

AK: Acanthamoeba keratitis; BAK: Benzalkonium chloride; CL: contact lens; CTC: 5-cyano-2,3-ditolyl-tetrazolium chloride; DES: Dry eye syndrome; PYG: Protease peptone-yeast extract-glucose

\section{Acknowledgements}

The authors are indebted to Sergio Llorens for his valuable technical assistance, and to Brian Crilly for his helpful revision of the manuscript.

\section{Funding}

This project was supported by Grant PI12/ 02725 from Instituto de Salud Carlos III (FISS) and FEDER by grant USPCEU-PC07/2013 of the Fundación Universitaria San Pablo CEU and by grant CEU-Banco de Santander. TSG was supported by Research Program EADS Construcciones Aeronáuticas S.A. Brasil I and by CAPES/Brazil (Grant BEX 9132/13-9).

\section{Availability of data and materials}

The datasets used and/or analyzed during the current study are available from the corresponding author upon reasonable request.

\section{Authors' contributions}

AM participated in the design of the laboratory techniques for viability measurement, participated in the data acquisition and research execution, analyzed and interpreted the data and was a major contributor in writing the manuscript. TSG participated in the design of the laboratory techniques for viability measurement (CTC staining), analyzed and interpreted the data and was a major contributor in writing the manuscript. CP and NGB participated in the data acquisition and research execution, analyzed and interpreted the data. CS and EC participated in the flow cytometry techniques by designing the protocols, acquiring the data and analyzing it. $\mathrm{Fl}$ participated in the design of the laboratory techniques for viability measurement, analyzed and interpreted the data and participated in the manuscript preparation. JMBC participated in the research design by choosing the artificial tears after analyzing the different preservatives. $\mathrm{CH}$ and CA participated in the research design, interpretation of the data and manuscript preparation. SF participated in the design of the laboratory techniques for viability measurement, participated in the data acquisition and research execution, analyzed and interpreted the data and was a major contributor in writing the manuscript. All authors read and approved the final manuscript.

Ethics approval and consent to participate

Not applicable.

\section{Consent for publication}

Not applicable.

\section{Competing interests}

The authors declare that they have no competing interests.

\section{Publisher's Note}

Springer Nature remains neutral with regard to jurisdictional claims in published maps and institutional affiliations.

\section{Author details}

${ }^{1}$ Facultad de Farmacia, Universidad San Pablo CEU, CEU Universities, Madrid, Spain. ${ }^{2}$ Facultad de Medicina, Universidad San Pablo CEU, CEU Universities, Madrid, Spain. ${ }^{3}$ Servicio de Oftalmología, Hospital Clínico San Carlos, Universidad Complutense, Madrid, Spain. ${ }^{4}$ Instituto de Salud Carlos III, Centro Nacional de Microbiología, WHO Collaborating Centre for Leishmaniasis, Madrid, Spain. ${ }^{5}$ CAPES Foundation, Ministry of Education of Brazil, Brasília, DF 70040-020, Brazil.

Received: 7 August 2017 Accepted: 11 January 2018

Published online: 22 January 2018

\section{References}

1. del Buey MA, Cristobal JA, Casas P, Goni P, Clavel A, Minguez E, et al. Evaluation of in vitro efficacy of combined riboflavin and ultraviolet a for Acanthamoeba isolates. Am J Ophthalmol. 2012;153(3):399-404.

2. Marciano-Cabral F, Cabral G. Acanthamoeba spp. as agents of disease in humans. Clin Microbiol Rev. 2003;16(2):273-307.

3. Yu HS, Choi KH, Kim HK, Kong HH, Chung DI. Genetic analyses of Acanthamoeba isolates from contact lens storage cases of students in Seoul, Korea. Kor J Parasitol. 2001;39(2):161-70.

4. Johnston SP, Sriram R, Qvarnstrom Y, Roy S, Verani J, Yoder J, et al. Resistance of Acanthamoeba cysts to disinfection in multiple contact lens solutions. J Clin Microbiol. 2009:47(7):2040-5.

5. Khan NA. Acanthamoeba: biology and increasing importance in human health. FEMS Microbiol Rev. 2006;30(4):564-95.

6. Lorenzo-Morales J, Khan NA, Walochnik J. An update on Acanthamoeba keratitis: diagnosis, pathogenesis and treatment. Parasite. 2015;22:10.

7. Page MA, Mathers WD. Acanthamoeba keratitis: a 12-year experience covering a wide spectrum of presentations, diagnoses, and outcomes. J Ophthalmol. 2013;2013:670242.

8. Panjwani N. Pathogenesis Of Acanthamoeba keratitis. Ocul Surf. 2010; 8(2):70-9.

9. Lorenzo-Morales J, Martin-Navarro CM, Lopez-Arencibia A, Arnalich-Montiel F, Pinero JE, Valladares B. Acanthamoeba keratitis: an emerging disease gathering importance worldwide? Trends Parasitol. 2013;29(4):181-7. 
10. Visvesvara GS, Moura H, Schuster FL. Pathogenic and Opportunistic free-living amoebae: Acanthamoeba spp., Balamuthia mandrillaris, Naegleria fowleri, and Sappinia diploidea. FEMS Immunol Med Microbiol. 2007;50(1):1-26.

11. Kilvington S, Gray T, Dart J, Morlet N, Beeching JR, Frazer DG, et al. Acanthamoeba keratitis: the role of domestic tap water contamination in the United Kingdom. Invest Ophthalmol Vis Sci. 2004;45(1):165-9.

12. McDonald M, Schachet JL, Lievens CW, Kern JR. Systane(R) ultra lubricant eye drops for treatment of contact lens-related dryness. Eye Contact Lens. 2014;40(2):106-10.

13. Fonn D. Targeting contact lens induced dryness and discomfort: what properties will make lenses more comfortable. Optom Vis Sci. 2007; 84(4):279-85.

14. Zeev MS, Miller DD, Latkany R. Diagnosis of dry eye disease and emerging technologies. Clin Ophthalmol. 2014;8:581-90.

15. Narayanan S, Redfern RL, Miller WL, Nichols KK, McDermott AM. Dry eye disease and microbial keratitis: is there a connection? Ocul Surf. 2013; 11(2):75-92.

16. Kymionis GD, Bouzoukis DI, Diakonis VF, Siganos C. Treatment of chronic dry eye: focus on cyclosporine. Clin Ophthalmol. 2008;2(4):829-36.

17. Moshirfar M, Pierson K, Hanamaikai K, Santiago-Caban L, Muthappan V Passi SF. Artificial tears potpourri: a literature review. Clin Ophthalmol. 2014:8:1419-33.

18. Magnet A, Galvan AL, Fenoy S, Izquierdo F, Rueda C, Fernandez Vadillo C, et al. Molecular characterization of Acanthamoeba isolated in water treatment plants and comparison with clinical isolates. Parasitol Res. 2012:111:383-92.

19. Caspers H, Page FC. An illustrated key to freshwater and soil amoebae with notes on cultivation and ecology. Ambleside, Cumbria: Freshwater Biological Association, Scientific Publication No. 34; 1976

20. Tu EY, Shoff ME, Gao W, Joslin CE. Effect of low concentrations of benzalkonium chloride on acanthamoebal survival and its potential impact on empirical therapy of infectious keratitis. JAMA Ophthalmol. 2013;131(5): 595-600.

21. Beattie TK, Seal DV, Tomlinson A, McFadyen AK, Grimason AM. Determination of amoebicidal activities of multipurpose contact lens solutions by using a most probable number enumeration technique. J Clin Microbiol. 2003:41(7):2992-3000.

22. Magnet A, Pardinas C, García de Blas N, DS Gomez T, Saavada C, Carrillo E, et al. Anti-Acanthamoeba activity of different artificial tears used in the treatment of amoeba keratitis. In: VII European Congress of Protistology. Seville: Federation of European Protistological Societies; 2015. p. 158. http:// www.semicrobiologia.org/protistologia/files/Book_of_Abstracts.pdf.

23. Kobayashi T, Mito T, Watanabe N, Suzuki T, Shiraishi A, Ohashi Y. Use of 5cyano-2,3-ditolyl-tetrazolium chloride staining as an indicator of biocidal activity in a rapid assay for anti-Acanthamoeba agents. J Clin Microbiol. 2012;50(5):1606-12.

24. Charnock C. Are multidose over-the-counter artificial tears adequately preserved? Cornea. 2006:25(4):432-7.

25. Aitken D, Hay J, Kinnear FB, Kirkness CM, Lee WR, Seal DV. Amebic keratitis in a wearer of disposable contact lenses due to a mixed Vahlkampfia and Hartmannella infection. Ophthalmology. 1996;103(3):485-94.

\section{Submit your next manuscript to BioMed Central and we will help you at every step:}

- We accept pre-submission inquiries

- Our selector tool helps you to find the most relevant journal

- We provide round the clock customer support

- Convenient online submission

- Thorough peer review

- Inclusion in PubMed and all major indexing services

- Maximum visibility for your research

Submit your manuscript at www.biomedcentral.com/submit

) Biomed Central 